\title{
NSGA-II Algorithm with a Local Search Strategy for Multiobjective Optimal Design of Dry-Type Air-Core Reactor
}

\author{
Chengfen Zhang ${ }^{1,2}$ and Xikui $\mathrm{Ma}^{1}$ \\ ${ }^{1}$ School of Electrical Engineering, Xian Jiaotong University, Xian 710049, China \\ ${ }^{2}$ College of Electrical Engineering, Henan University of Technology, Zhengzhou 450001, China \\ Correspondence should be addressed to Chengfen Zhang; eegszhangcf@126.com
}

Received 9 January 2015; Revised 8 March 2015; Accepted 9 March 2015

Academic Editor: Muhammad N. Akram

Copyright (c) 2015 C. Zhang and X. Ma. This is an open access article distributed under the Creative Commons Attribution License, which permits unrestricted use, distribution, and reproduction in any medium, provided the original work is properly cited.

\begin{abstract}
Dry-type air-core reactor is now widely applied in electrical power distribution systems, for which the optimization design is a crucial issue. In the optimization design problem of dry-type air-core reactor, the objectives of minimizing the production cost and minimizing the operation cost are both important. In this paper, a multiobjective optimal model is established considering simultaneously the two objectives of minimizing the production cost and minimizing the operation cost. To solve the multiobjective optimization problem, a memetic evolutionary algorithm is proposed, which combines elitist nondominated sorting genetic algorithm version II (NSGA-II) with a local search strategy based on the covariance matrix adaptation evolution strategy (CMA-ES). NSGA-II can provide decision maker with flexible choices among the different trade-off solutions, while the localsearch strategy, which is applied to nondominated individuals randomly selected from the current population in a given generation and quantity, can accelerate the convergence speed. Furthermore, another modification is that an external archive is set in the proposed algorithm for increasing the evolutionary efficiency. The proposed algorithm is tested on a dry-type air-core reactor made of rectangular cross-section litz-wire. Simulation results show that the proposed algorithm has high efficiency and it converges to a better Pareto front.
\end{abstract}

\section{Introduction}

As an important apparatus applied to harmonic filtering, short circuit current limiting, and reactive power compensation, dry-type air-core reactor plays a vital role in reducing failure and improving security of power system operation. In the past two decades, the design problem for reducing the production cost and the operation cost of dry-type air-core reactor has received considerable attention. Most literatures about the design problem of dry-type air-core reactors are mainly focused on the following aspects: (1) the fast and accurate computation of the inductance, magnetic field, and winding power loss [1-4]; (2) the complete model for impulse voltage distribution and electrical strength $[5,6]$; and (3) acoustic noise evaluation or controls $[7,8]$. Up to now, however, only a few works have been published on the optimal design problem of dry-type air-core reactor. For example, Liu et al. [9] employed the genetic algorithm to minimize the operation cost measured by the power loss of dry-type air-core reactor, and obtained the same results as the traditional design method only. In [10], based on the balance of the additional equality constraint conditions, a hybrid method of genetic algorithm and simplex method is proposed for the optimum design of the round-wire dry-type air-core reactor to enhance the local searching ability and improve the optimization efficiency. Unfortunately, the optimum design of dry-type air-core reactor is considered as a single objective problem with several constraints, although it involves more than one objective.

Actually, it is well known that the optimum design of drytype air-core reactor is a very complex problem as it requires the simultaneous minimization of two objective functions. The first objective deals with the minimization of the production cost by reducing aluminum wire weight. The second objective minimizes the operation cost by the minimization of power loss. And the voltage, height, package temperature rise and current density may be considered as constraint conditions. In theory, these two objectives are in conflict with 
each other. No single solution can be claimed as an optimum solution to multiple conflicting objectives, the resulting multiobjective optimization problem involves a number of tradeoff solutions. In order to provide a means to assess trade-off between two conflicting objectives, one way is to formulate the optimum design of dry-type air-core reactor as a multiobjective optimization problem.

This paper is concerned with the optimum design of drytype air-core reactor made of rectangular cross-section litzwire as a multiobjective optimization problem. Due to the nondifferentiality, nonlinearity, high-constraint, and highdiscretion of the optimum design problem, most traditional optimization or search approaches (e.g., Sequential Search, Tabu Search, etc.) not only often fall into local maxima and minima, but also are not easily applied to solve the optimization problem with multiobjectives. As one of the elegant approaches, the elitist nondominated sorting genetic algorithm II (NSGA-II) [11] has been demonstrated to be one of the efficient algorithms for solving multiobjective optimization problems such as generation expansion planning problem $[12,13]$, economic and environmental power dispatch [14], multipurpose water reservoir management [15], and the periodic operation of the naphtha pyrolysis process [16]. Although NSGA-II is a powerful algorithm that captures a global search space and obtains well-distributed Pareto front, it is very time-consuming to solve the complex design problem; that is, it suffers from the lower convergence speed, especially at the early stage of the optimization. Therefore, it is of great importance to enhance the algorithm's search ability. One of our aims is to makes use of a local search strategy to speed up the convergence of the NSGA-II.

In this study, the NSGA-II algorithm with certain modifications is applied to settle the multiobjective problem. As an approach to improve the NSGA-II algorithm, a local search strategy based on the Covariance Matrix Adaptation Evolution Strategy (CMA-ES) [17] is incorporated into the original NSGA-II algorithm to promote algorithm's exploiting capability and then to accelerate the algorithm convergence for the multiobjective optimum design of dry-type air-core reactor made of rectangular cross-section litz-wire, hereafter designated as the NSGA-CMA algorithm. In practice, the local search is applied to the certain selected nondominated individuals of a given generation. Furthermore, an external archive is set in the proposed algorithm for improving the evolutionary efficiency especially at the early stage. To validate the performance of the proposed algorithm, the performance measures of the convergence metric and the two set coverage measure are considered. The effectiveness of the proposed algorithm is tested on a $50 \mathrm{kVar}$ (i.e., $317.5 \mathrm{~V}, 157.5 \mathrm{~A}$, $6.42 \mathrm{mH}$ ) dry-type air-core reactor. The results show that the proposed algorithm not only converges to a better Pareto front, but also is of very high efficiency.

The paper is organized as follows. The optimum design of dry-type air-core reactor is formulated as a multiobjective optimization problem with a number of equality constraints and inequality constraints in Section 2. Then, the details of the proposed NSGA-CMA is described in Section 3 and its implementation to the optimum design of dry-type air-core reactor is outlined in Section 4, the experiment results and

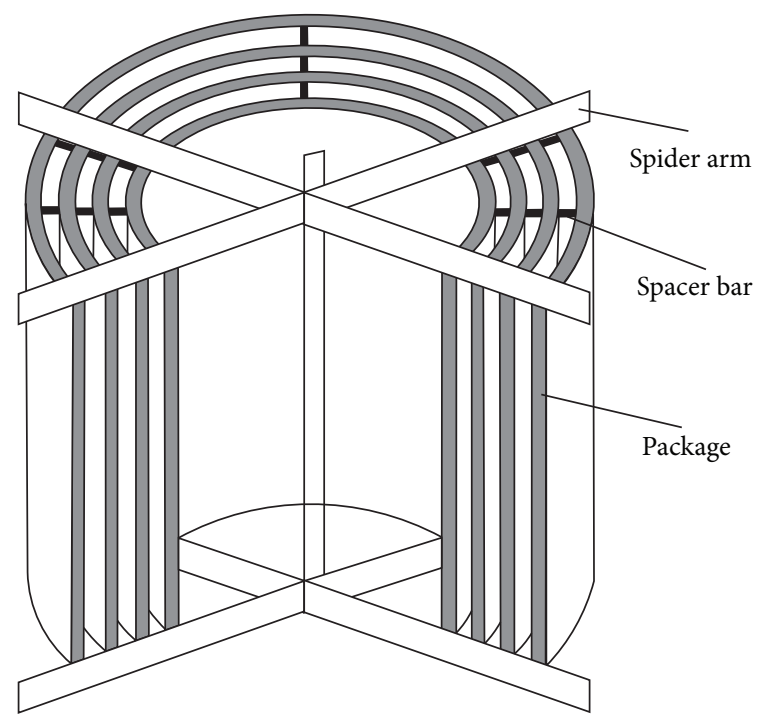

FIGURE 1: Diagram of dry-type air-core reactor.

the comparisons with the original NSGA-II are also presented. Finally, some conclusions are drawn in Section 5.

\section{Problem Formulation}

The dry-type air-core reactor, the diagram of which is shown in Figure 1, consists of several parallel packages; adjacent packages are connected by spacer bars; each package is composed of parallel multilayer coil windings; both ends of the coil windings are welded in spider frame made of aluminum. In this paper, four mutually independent variable parameters defining the structure of dry-type air-core reactor are subjected to optimization. The design variables are the number of winding package $N_{p}$, the number of winding layer $N_{c}$, the inner diameter $D_{\text {in }}$, and the average side length of litzwire $l_{\mathrm{av}}$. In these design variables, the inner diameter $D_{\text {in }}$ and the average side length $l_{\mathrm{av}}$ are taken as continuous variables, whereas the number of winding package $N_{p}$ and the number of winding layer $N_{c}$ are taken as discrete values.

The optimal design of dry-type air-core reactor is equivalent to finding a set of best decision vectors that minimizes the two competing objective functions, the production, and the operation cost, subject to a number of equality constraints and inequality constraints. Apparently, the multiobjective formulation is a choice way to treat the nonlinear and constrained optimal problem. Its mathematical model can be described as follows.

2.1. The Objective Functions. Since minimizing the aluminum wire weight is equivalent to minimizing the production cost, the first objective function is to minimize the aluminum wire weight, usually defined as

$$
\min W_{\mathrm{AL}}=\pi \rho \sum_{i=1}^{N_{c}} A_{i} B_{i} D_{i} w_{i},
$$

where $W_{\mathrm{AL}}$ is the aluminum wire weight, $\rho$ the mass density of aluminum, $A_{i}$ and $B_{i}$ the radial-side length and the axial-side 
length of the $i$ th layer litz-wire, respectively, $D_{i}$ the middle diameter (the average value of inner diameter and outer diameter) of the $i$ th layer litz-wire, $w_{i}$ the turns of the $i$ th layer litz-wire.

It is well known that the smaller power loss results in the less operation cost, so the second objective function can be represented by the following expression:

$$
\begin{aligned}
\min P_{\text {Loss }}= & \frac{\pi}{\gamma} \sum_{i=1}^{N_{c}} \frac{D_{i} w_{i} I_{i}^{2}}{A_{i} B_{i}}+\frac{\pi \gamma \omega^{2}}{12} \\
& \cdot \sum_{i=1}^{N_{c}} \sum_{j=1}^{w_{i}} D_{i} A_{i} B_{i}\left(A_{i}^{2} B_{z_{i j}}^{2}+B_{i}^{2} B_{r_{i j}}^{2}\right),
\end{aligned}
$$

where $P_{\text {Loss }}$ is the power loss, $\gamma$ the conductivity of aluminum, $I_{i}$ the current through the $i$ th layer litz-wire, $\omega$ the angular frequency, $B_{z_{i j}}$ the magnetic flux density's axial component of the $i$ th layer litz-wire at the $j$ th turn, and $B_{r_{i j}}$ the magnetic flux density's radial component of the $i$ th layer litz-wire at the $j$ th turn.

2.2. Design Constraints. The objective functions are subjected to following some equality constraints and inequality constraints.

2.2.1. Equality Constraints. In order to design a high performance dry-type air-core reactor, some additional equality constraints are proposed. There are two most common additional constraints which are layer resistance drop balance constraint and package temperature rise balance constraint. Layer resistance drop balance can assure that the air-core reactor has the minimum power loss; package temperature rise balance can assure the best heat sinking effect. In addition, in order to make the air-core reactor have more compact structure and reduce the manufacturing cost, the package height balance constraint should be considered too. The three additional equality constraints are expressed as follows.

(1) Layer resistance drop balance constraint:

$$
\frac{D_{i} w_{i} I_{i}}{A_{i} B_{i}}=\frac{D_{j} w_{j} I_{j}}{A_{j} B_{j}}, \quad i \neq j, i, j=1, \ldots, N_{c} .
$$

(2) Package height balance constraint:

$$
H_{i}=H_{j}, \quad i \neq j, i, j=1, \ldots, N_{p},
$$

where $H_{i}$ is the height of the $i$ th package.

(3) Package temperature rise balance constraint:

$$
T_{i}=T_{0}, \quad i=1, \ldots, N_{p},
$$

where $T_{0}$ is the expectant degree of users, $T_{i}$ is the temperature rise of the $i$ th package and is defined as

$$
T_{i}=0.432 H_{i}^{0.2} q_{i}^{0.8}, \quad i=1, \ldots, N_{p}
$$

where $q_{i}$ is unit area heat load of the $i$ th package.

\subsubsection{Inequality Constraints}

(1) Current Density Limits. For stable operation, the current density of each layer litz-wire must fail into a region of operation defined by lower and upper limits:

$$
J^{\min } \leq J_{i} \leq J^{\max }, \quad i=1, \ldots, N_{c},
$$

where $J_{i}$ is the current density of the $i$ th layer litz-wire and $J^{\mathrm{min}}$ and $J^{\mathrm{max}}$ are the lower and the upper limits of the current density, respectively. And $J_{i}$ is defined as follows:

$$
J_{i}=\frac{I_{i}}{A_{i} B_{i}}, \quad 1 \leq i \leq N_{c} .
$$

(2) Design Variable Limits. The limits on these design variables are given by the following inequalities:

$$
\begin{aligned}
N_{p}^{\mathrm{min}} & \leq N_{p} \leq N_{p}^{\max }, \\
3 N_{p} & \leq N_{c} \leq 5 N_{p}, \\
D_{\mathrm{in}}^{\min } & \leq D_{\mathrm{in}} \leq D_{\mathrm{in}}^{\max }, \\
l_{\mathrm{av}}^{\mathrm{min}} & \leq l_{\mathrm{av}} \leq l_{\mathrm{av}}^{\max },
\end{aligned}
$$

where the superscripts "min" and "max" denote the corresponding lower and upper limits, respectively, $l_{\mathrm{av}}$ is defined as

$$
l_{\mathrm{av}}=\sqrt{\frac{1}{N_{c}} \sum_{i=1}^{N_{c}} A_{i} B_{i}}
$$

It is now evident from the above discussion that the optimal design problem of dry-type air-core reactor is a nonlinear engineering optimization problem without analytical expression, that is, the objective functions cannot be expressed as the functions of the design variables in analytical form. However, the variable values in the objective functions can be obtained from the above four mutually independent design variables in the process of design.

\section{The Proposed NSGA-CMA Algorithm}

3.1. Analysis of NSGA-II Algorithm. NSGA-II algorithm has been demonstrated as one of the most efficient and famous algorithms for multiobjective optimization. It uses the fast nondominated sorting to rank the population fronts and a parameter called crowding distance is calculated in the same front. Then, tournament selection is made between two individuals randomly selected from parent population. The individual with lower front number is selected if the two individuals come from different fronts. The individual with higher crowding distance is selected if the two individuals are from the same front. Then, both the crossover and the mutation operators are used to generate a new offspring population. Finally, the parent and offspring populations are combined together where a fast nondominated sorting and crowding 
distance assignment procedure is used to rank the combined population and only the best $N$ individuals are selected as the new parent population.

NSGA-II is proposed on basis of nondominated sorting genetic algorithm (NSGA) [18], and the main advantages of NSGA-II compared with NSGA are as follows: (1) it uses a fast nondominated sorting approach, (2) it has no sharing parameter, and (3) it uses an elitist strategy. NSGA-II has good global search ability and well distributed nondominated solutions in the Pareto front. But the convergence capability of NSGA-II is limited when solving the engineering optimal problem such as the multiobjective optimum design of drytype air-core reactor, and its elite strategy may result in the loss of the nondominated solutions in the Pareto front.

Memetic algorithms perform global exploration by evolutionary algorithms and local exploitation by a local search strategy, respectively. It has global search ability of evolutionary algorithm and local search ability of local search strategy simultaneously. It is reported that memetic algorithm converges to high quality solutions more efficiently than evolution algorithms $[19,20]$. To improve the efficiency of NSGAII, in this paper, a local search strategy based on the CMA-ES is introduced into the algorithm to accelerate the speed of convergence. In addition, an external archive is set in NSGAII to increase the evolutionary efficiency.

3.2. Local Search Strategy Based on the CMA-ES. Local search can enhance the search capability of evolutionary algorithms by carrying out local exploitation, and the global and local searches may be well balanced. The CMA-ES is probably one of the most powerful self-adaptation mechanisms for continuous search spaces. It uses a covariance matrix to construct the mutation distribution and adapts this covariance matrix from cumulative paths of successful mutations. Firstly, it samples a number of new candidate solutions from a multivariate normal distribution and then updates the distribution by means of two major strategies: step size update and covariance matrix adaptation. The process in five steps is given and explained as follows.

Step 1. Initialize $g \in N, x_{1}^{(g)}, \ldots, x_{\lambda}^{(g)} \in R^{n}, C^{(g)} \in R^{n \times n}$, $p_{c}^{(g)} \in R^{n}, p_{\sigma}^{(g)} \in R^{n}$ and $\sigma^{(g)} \in R^{+}$as follows.

$$
\begin{aligned}
& g=0, p_{c}^{(0)}=p_{\sigma}^{(0)}=0, C^{(0)}=I \text { (unity matrix). } \sigma^{(0)} \\
& \text { and } x_{1}^{(0)}, \ldots, x_{\lambda}^{(0)} \text { are given as inputs, }
\end{aligned}
$$

where $g$ is the generation counter and the superscript " $(g)$ " represents the $g$ th generation, $n$ is the dimension of searching space and also the number of the variable for local search, $\lambda$ is the population size, $x_{k}^{(g)}$ is the object parameter vector of the $k$ th individual, $C^{(g)}$ is the $n \times n$ covariance matrix, $p_{c}^{(g)}$ is the evolution path used to speed up the adaptation of the covariance matrix $C^{(g)}, p_{\sigma}^{(g)}$ is also the evolution path that is similar to $p_{c}^{(g)}$, and $\sigma^{(g)}$ is the global step size.
Step 2. At each generation $g+1$, compute the object parameter vector $x_{k}^{(g+1)}$ as follows:

$$
\begin{aligned}
& x_{k}^{(g+1)}=\langle x\rangle_{w}^{(g)}+\sigma^{(g)} \underbrace{B^{(g)} D^{(g)} z_{k}^{(g+1)}}_{\sim N\left(0, C^{(g)}\right)}, \quad k=1, \ldots, \lambda, \\
& \langle x\rangle_{w}^{(g)}=\frac{1}{\sum_{i=1}^{\mu} \omega_{i}} \sum_{i=1}^{\mu} \omega_{i} x_{i: \lambda}^{(g)}, \quad \omega_{i} \in R^{+},
\end{aligned}
$$

where $\mu$ is the number of the selected search points in the population; $\langle x\rangle_{w}^{(g)}$ represents the weighted mean of the $\mu$ best individuals of generation $g$; $\omega_{i}$ is the weight coefficient; the subscript $i: \lambda$ denotes the $i$ th best individual; $z_{k}^{(g+1)}$ is the $N(0, I)$ distributed random vector of the $k$ th individual; the components of $z_{k}^{(g+1)}$ are independent $N(0,1)$ distribution; $C^{(g)}$ determines $B^{(g)}$ and $D^{(g)}$; the singular value decomposition of $C^{(g)}$ can be expressed as $C^{(g)}=B^{(g)} D^{(g)}\left(B^{(g)} D^{(g)}\right)^{T}=$ $B^{(g)}\left(D^{(g)}\right)^{2}\left(B^{(g)}\right)^{T}$.

Step 3. Update the covariance matrix $C^{(g)}$ by means of the evolution path $p_{c}^{(g+1)}$ :

$$
\begin{aligned}
& p_{c}^{(g+1)}=\left(1-c_{c}\right) p_{c}^{(g)}+\sqrt{c_{c}\left(2-c_{c}\right)} \cdot c_{w} B^{(g)} D^{(g)}\langle z\rangle_{w}^{(g+1)}, \\
& C^{(g+1)}=\left(1-c_{\text {cov }}\right) C^{(g)}+c_{\text {cov }} p_{c}^{(g+1)}\left(p_{c}^{(g+1)}\right)^{T},
\end{aligned}
$$

where $c_{c} \in[0,1]$ determines the cumulative time for $p_{c}^{(g+1)}$, $c_{\text {cov }} \in[0,1]$ is the change rate of $C^{(g)}$, and the expressions of $c_{w}$ and $\langle z\rangle_{w}^{(g+1)}$ are given as follows:

$$
\begin{gathered}
c_{w}=\frac{\sum_{i=1}^{\mu} \omega_{i}}{\sqrt{\sum_{i=1}^{\mu} \omega_{i}^{2}}}, \\
\langle z\rangle_{w}^{(g+1)}=\frac{1}{\sum_{i=1}^{\mu} \omega_{i}} \sum_{i=1}^{\mu} \omega_{i} z_{i: \lambda}^{(g+1)},
\end{gathered}
$$

respectively.

Step 4. Update the global step size $\sigma$ by using the evolution path $p_{\sigma}^{(g+1)}$ :

$$
\begin{aligned}
& p_{\sigma}^{(g+1)}=\left(1-c_{\sigma}\right) p_{\sigma}^{(g)}+\sqrt{c_{\sigma}\left(2-c_{\sigma}\right)} \cdot c_{w} B^{(g)}\langle z\rangle_{w}^{(g+1)}, \\
& \sigma^{(g+1)}=\sigma^{(g)} \exp \left(\frac{1}{d_{\sigma}} \frac{\left\|p_{\sigma}^{(g+1)}\right\|-\widehat{\chi}_{n}}{\hat{\chi}_{n}}\right),
\end{aligned}
$$

where $c_{\sigma} \in[0,1]$ determines the cumulative time for $p_{\sigma}^{(g+1)}$; $d_{\sigma} \geq 1$ determines the possible change rate of $\sigma^{(g)} ; \widehat{\chi}_{n}$ is the expectation of the length of the $N(0,1)$ distributed random vector, which is approximated by $\widehat{\chi}_{n} \approx \sqrt{n}\left(1-1 / 4 n+1 / 21 n^{2}\right)$.

Step 5. The computation is repeated until the maximum number of generations is met. 
It should be pointed out that the local search strategy based on CMA-ES cannot be directly used to handle multiple objectives. To apply the local search strategy based on CMAES to the constrained two-objective optimization problem in this paper, the following overall objective $F$ is calculated by linear combination of different objectives as a weighted sum:

$$
F=d_{1} W_{\mathrm{AL}}+d_{2} P_{\text {Loss }}+V,
$$

where $d_{1}$ and $d_{2}$ are the weight coefficients and $d_{1}+d_{2}=$ 1 and $V$ is the constraint violation value used as the penalty function. Here, to avoid priority-based weight, the values of the two weight coefficients are set to be equal, that is, $d_{1}=$ $d_{2}=0.5$, and the normalized objective function values are to be used.

Although the local search strategy may improve the performance of the original NSGA-II, it implies an additional cost, which may become prohibitive in the optimization design of dry-type air-core reactor. When the quality of an individual is very poor, the application of local search strategy also seems to be waste of the computing time. Thus, the local search (i.e., CMA-ES) is applied only to nondominated individuals randomly selected from the current population obtained from elitism of a given generation. Actually, our experience has shown that in dry-type air-core reactor the numbers of winding package and winding layer have a great influence upon the results. Therefore, in order to get good search results, the local search is applied only to the inner diameter and average side length.

Since the overall objective function is used in the CMAES method, the points obtained from the CMA-ES should be checked for nondominance before they are accepted in the current population. If the point obtained by the CMA-ES dominates any point in the current population, the dominated point must be replaced by the new point.

3.3. External Archive. In each generation of the original NSGA-II, a new offspring population is generated. The parent and offspring populations are combined together where a fast nondominated sorting procedure is used to sort the combined population and only the best $N$ individuals are selected.

In order to increase the evolutionary efficiency especially at the early generation, an external archive of size $M$ is introduced into NSGA-II, and individuals of the archive participate in the tournament selection together with individuals of the parent population. Individuals in the first front of the combined population are stored in the archive, when the archive exceeds the maximal size $M$, the individuals with the lowest crowding distance are to be discarded.

3.4. Procedures of the Proposed Algorithm. Let gen and $G_{\max }$ denote the generation number and the maximum generation number, respectively. The local search is applied to $N_{\mathrm{CMA}}$ nondominated individuals each $s$ generation. The application of NSGA-CMA algorithm to the multiobjective optimization design of dry-type air-core reactor can be written as follows. The corresponding flowchart is given in Figure 2.
Step 1 (initialization). Input the initial parameters of the $50 \mathrm{kVar}$ dry-type air-core reactor and the proposed algorithm; randomly generate an initial population; set gen $=1$.

Step 2 (evaluation). Calculate the values of the objective functions and constraints for each individual in the current population.

Step 3 (fast nondominated sorting and crowding distance assignment). Apply nondominated sorting to classify the current population into different nondominated fronts and then calculate the crowding distance of each individual in the same front.

Step 4 (selection). Perform tournament selection between two individuals randomly selected from the parent population and external archive.

Step 5 (crossover and mutation). Apply a crossover operator to each of the randomly selected $N / 2$ pairs of parent individuals and a new population is generated. Then, apply a mutation operator to the new population and a new offspring population is obtained.

Step 6 (elitist strategy). Combine parent and offspring population to generate a combined population and then apply fast nondominated sorting and crowding distance assignment to the combined population. Select $N$ best individuals as a new parent population according to nondominated front and crowding distance.

Step 7 (local search). If $\bmod ($ gen, $s)=0$, apply local search strategy based on CMA-ES to $N_{\text {CMA }}$ nondominated individuals randomly selected from the new parent population. Then, update the new parent population. Otherwise, go to Step 8.

Step 8 (external archive). In the new generated parent population, individuals in the first nondominated front are stored in the external archive. When the archive exceeds the maximal size $M$, individuals with lower crowding distance are to be discarded.

Step 9 (termination). If gen $>G_{\max }$, end the algorithm. Otherwise, return to Step 2.

\section{Test Results and Discussion}

To verify the effectiveness of the proposed algorithm, NSGAII and NSGA-CMA are used for the optimization design of a $50 \mathrm{kVar}$ (i.e., $317.5 \mathrm{~V}, 157.5 \mathrm{~A}$, and $6.42 \mathrm{mH}$ ) dry-type aircore reactor made of rectangular cross-section litz-wire, and comparisons are made between their results.

4.1. Mixed Variable Handling Method. There are four mixed design variables in the optimization design of dry-type aircore reactor. The number of winding package $N_{p}$ and the number of winding layer $N_{c}$ are integer variables and the inner diameter $D_{\text {in }}$ and the average side length of the litz-wire $l_{\mathrm{av}}$ are continuous variables. Here, the hybrid code is used, 
TABLE 1: Parameters for a 50 kVar dry-type air-core reactor.

\begin{tabular}{lccccccccc}
\hline Parameters & $N_{p}^{\min }$ & $N_{p}^{\max }$ & $D_{\mathrm{in}}^{\min }(\mathrm{mm})$ & $D_{\mathrm{in}}^{\min }(\mathrm{mm})$ & $l_{\mathrm{av}}^{\min }(\mathrm{mm})$ & $l_{\mathrm{av}}^{\max }(\mathrm{mm})$ & $J^{\min }\left(\mathrm{A} / \mathrm{mm}^{2}\right)$ & $J^{\max }\left(\mathrm{A} / \mathrm{mm}^{2}\right)$ & $T_{0}\left({ }^{\circ} \mathrm{C}\right)$ \\
\hline Values & 2 & 7 & 400 & 1000 & 2 & 4 & 0.9 & 1.5 & 75 \\
\hline
\end{tabular}

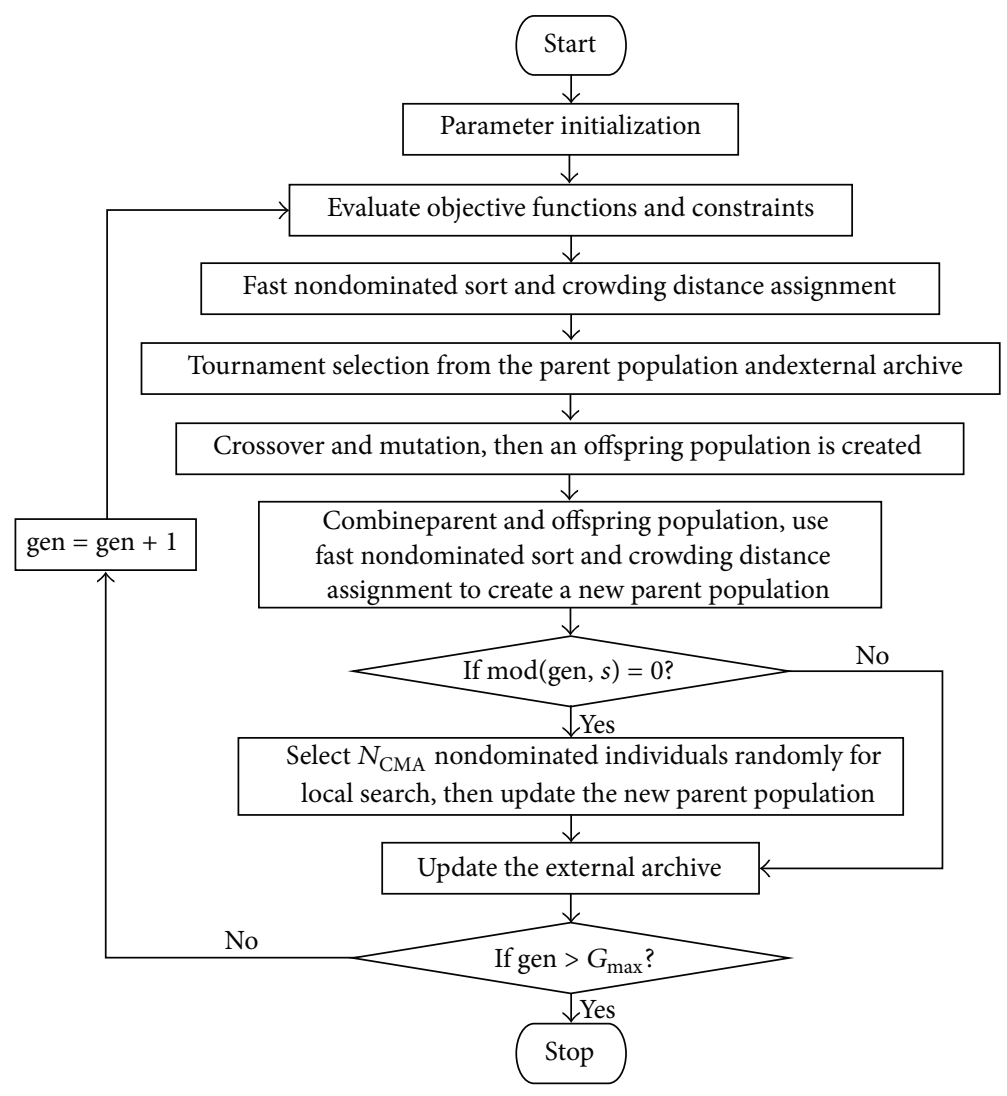

FIGURE 2: Flowchart of NSGA-CMA algorithm.

that is, the binary code for the integer variables and the real code for the continuous variables.

4.2. Parameter Settings. The main parameters of a $50 \mathrm{kVar}$ dry-type air-core reactor are the extreme values of design variables and the constraint conditions, which are given in Table 1.

The parameter settings of CMA-ES, which are discussed in [17], are summarized in Table 2. The parameters $\lambda$ and $\mu$ are comparatively uncritical and can be chosen in a wide range.

In the proposed algorithm, the crossover probability is denoted as $P_{c}$, the mutation probabilities as $P_{m}$ for binary code and as $P_{\text {mreal }}$ for real code; the distribution indexes are expressed as $\eta_{c}$ for crossover operator and as $\eta_{m}$ for mutation operator. In the local search procedure, let $G_{\mathrm{CMA}}$ denote the number of generation for local search and $\delta_{D_{\mathrm{in}}}$ and $\delta_{l_{\mathrm{av}}}$ the neighborhood size of the inner diameter and the average side length of the selected nondominated individuals, respectively. The parameters for NSGA-CMA are given in Table 3. Here, the parameters for NSGA-II are set at the same values as NSGA-CMA to ensure the comparison is fair.
4.3. Performance Measurements. In order to evaluate the performance of the proposed algorithm, the convergence metric [21] and the two set coverage measure [22] are used as evaluation indexes.

The convergence metric measures the extent of convergence towards a reference set, and lower values of the metric represent good convergence ability. Let $P^{(t)}(t=1, \ldots$, gen) be the population of the $t$ th generation, $P^{*}$ the reference or target set, in which the points can be either a set of Pareto optimal points (if known) or the nondominated set of points in a combined pool of all generation-wise populations, and $A$ the final approximate Pareto optimal set obtained from $P^{(t)}$. Then, the smallest normalized Euclidean distance to $P^{*}$ can be calculated from each point $i$ in $A$ as follows:

$$
p d_{i}=\min _{j=1}^{\left|P^{*}\right|} \sqrt{\sum_{k=1}^{m}\left(\frac{f_{k}(i)-f_{k}(j)}{f_{k}^{\max }-f_{k}^{\min }}\right)^{2}}
$$

where $m$ is the number of objective functions, $f_{k}(i)$ is the $k$ th objective function value of individual $i, f_{k}^{\max }$ and $f_{k}^{\min }$ are the maximum and minimum values of the $k$ th objective 
TABLE 2: Default parameter settings for CMA-ES.

\begin{tabular}{lcccccc}
\hline Parameters & $\lambda$ & $\mu$ & $\omega_{i=1, \ldots, \mu}$ & $c_{c}$ & $c_{\mathrm{cov}}$ & $c_{\sigma}$ \\
\hline Values & $4+[3 \ln (n)]$ & {$\left[\frac{\lambda}{2}\right]$} & $\ln \left(\frac{\lambda+1}{2}\right)-\ln (i)$ & $\frac{4}{n+4}$ & $\frac{2}{(n+\sqrt{2})^{2}}$ & $\frac{4}{n+4}$ \\
\hline
\end{tabular}

TABle 3: Parameters for NSGA-CMA.

\begin{tabular}{lccccccccccccccccc}
\hline Parameters & $N$ & $M$ & $G_{\max }$ & $P_{c}$ & $P_{m}$ & $P_{\text {mreal }}$ & $\eta_{c}$ & $\eta_{m}$ & $N_{\mathrm{CMA}}$ & $G_{\mathrm{CMA}}$ & $s$ & $n$ & $\lambda$ & $\mu$ & $\delta_{D_{\mathrm{in}}}(\mathrm{mm})$ & $\delta_{l_{\mathrm{av}}}(\mathrm{mm})$ \\
\hline Values & 100 & 100 & 100 & 0.9 & 0.01 & 0.5 & 20 & 20 & 5 & 4 & 10 & 2 & 5 & 3 & 70 & & 0.1 \\
\hline
\end{tabular}

function in $P^{*}$, respectively. The convergence metric is the average value of the normalized distance for all points in $A$ :

$$
C\left(P^{(t)}\right)=\frac{\sum_{i=1}^{|A|} p d_{i}}{|A|}
$$

To keep the convergence metric within $[0,1]$, we normalize the $C\left(P^{(t)}\right)$ values according to $\bar{C}\left(P^{(t)}\right)=C\left(P^{(t)}\right) / C\left(P^{(0)}\right)$.

Let $P^{\prime}$ and $P^{\prime \prime}$ be two approximate Pareto optimal sets. The two-set coverage measure is defined as

$$
\operatorname{CS}\left(P^{\prime}, P^{\prime \prime}\right)=\frac{\left|\left\{a^{\prime \prime} \in P^{\prime \prime} ; \exists a^{\prime} \in P^{\prime}: a^{\prime} \preceq a^{\prime \prime}\right\}\right|}{\left|P^{\prime \prime}\right|}
$$

where $\preceq$ means dominate or equal (also called weakly dominate); $a^{\prime}$ and $a^{\prime \prime}$ are the elements of $P^{\prime}$ and $P^{\prime \prime}$, respectively. The value $\operatorname{CS}\left(P^{\prime}, P^{\prime \prime}\right)=1$ means that all the elements of $P^{\prime \prime}$ are weakly dominated by $P^{\prime} \cdot \operatorname{CS}\left(P^{\prime}, P^{\prime \prime}\right)=0$ implies that no element of $P^{\prime \prime}$ is weakly dominated by $P^{\prime}$. Since the two set coverage measure is not always symmetric, both values $\operatorname{CS}\left(P^{\prime}, P^{\prime \prime}\right)$ and $\operatorname{CS}\left(P^{\prime \prime}, P^{\prime}\right)$ have to be considered.

4.4. Results and Discussion. To compare the proposed algorithm with NSGA-II, the two algorithms run for 20 times, respectively, with 20 initial populations of random solutions (one for each run). For the optimization problem discussed in this paper, there is no known true Pareto front, so a reference set is used to calculate the performance metric. The reference set represented as $P^{*}$ is obtained by adopting all the Pareto optimal solutions from all of the 40 runs.

To demonstrate the effectiveness of the algorithm, the parameter function evaluation is used, and its budget is set to 10000. Since the function evaluation always consumes most of the time of the algorithm for the engineering optimization problems, and the overall number of function evaluations is high.

Figure 3 shows the changes in the mean values of the convergence metric $\bar{C}$ of NSGA-II and NSGA-CMA versus the number of function evaluation. It can be seen that the average convergence metric values of the two algorithms are similar in the early iterative process. The average convergence metric values of NSGA-CMA are significantly decreased when the local search strategy based on CMA-ES is performed. After that, the average convergence metric values of the two algorithms are all decreased slowly, and the convergence metric values of NSGA-CMA are always smaller than

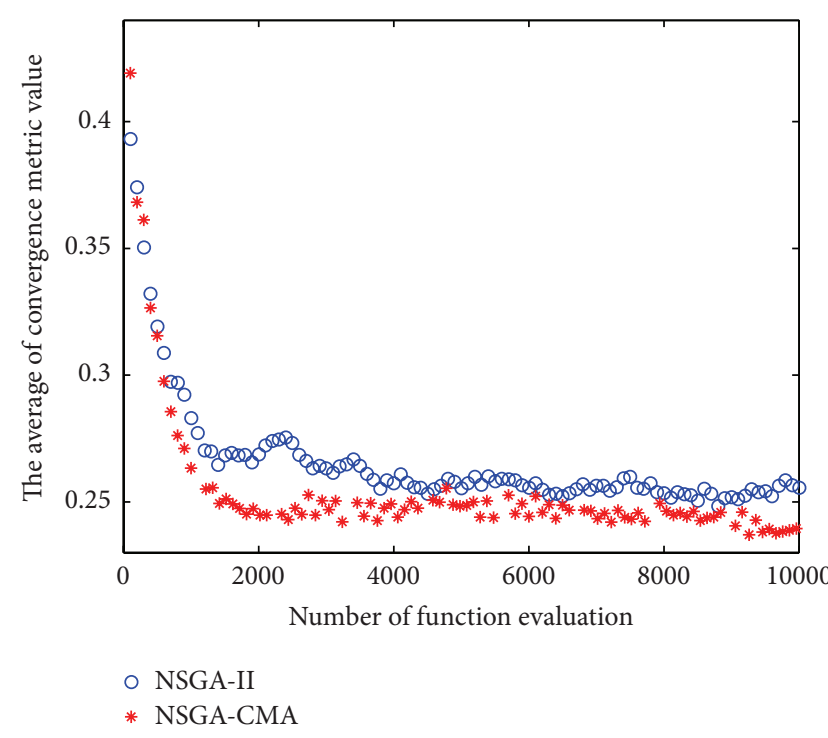

FIgURE 3: Curve of average convergence metric with 10000 function evaluations.

those of NSGA-II algorithm. It means that the nondominated solutions obtained by NSGA-CMA are obviously much more converged to the reference set than the original NSGA-II especially in early generation.

Figure 4 presents the mean values of the two set coverage metric $\operatorname{CS}\left(P^{\prime}, P^{\prime \prime}\right)$ and $\operatorname{CS}\left(P^{\prime \prime}, P^{\prime}\right)$ against the number of function evaluation; here $P^{\prime}$ and $P^{\prime \prime}$ are the Pareto optimal sets of NSGA-II and NSGA-CMA, respectively. It is seen that the two set coverage metrics $\operatorname{CS}\left(P^{\prime}, P^{\prime \prime}\right)$ and $\operatorname{CS}\left(P^{\prime \prime}, P^{\prime}\right)$ are similar in the initial stage, $\operatorname{CS}\left(P^{\prime \prime}, P^{\prime}\right)$ is increased evidently when the local search strategy is performed, then the two set coverage metrics $\operatorname{CS}\left(P^{\prime}, P^{\prime \prime}\right)$ and $\operatorname{CS}\left(P^{\prime \prime}, P^{\prime}\right)$ decrease gradually and $\operatorname{CS}\left(P^{\prime}, P^{\prime \prime}\right)$ is always lower than $\operatorname{CS}\left(P^{\prime \prime}, P^{\prime}\right)$. It means that NSGA-CMA is more efficient than the original NSGA-II, and it has more nondominated solutions which dominate the nondominated solutions obtained by NSGA-II.

In order to intuitively compare the distribution of the solutions obtained by the two algorithms, Figures 5 and 6 show the Pareto fronts obtained from the two algorithms at the 2500th and 10000th function evaluations, respectively. It can be seen from Figure 5 that all the solutions do not convergence to the reference set $P^{*}$ at this time, but the Pareto optimal solutions obtained by NSGA-CMA are more close to the reference set $P^{*}$. It can be seen from Figure 6 that the Pareto 


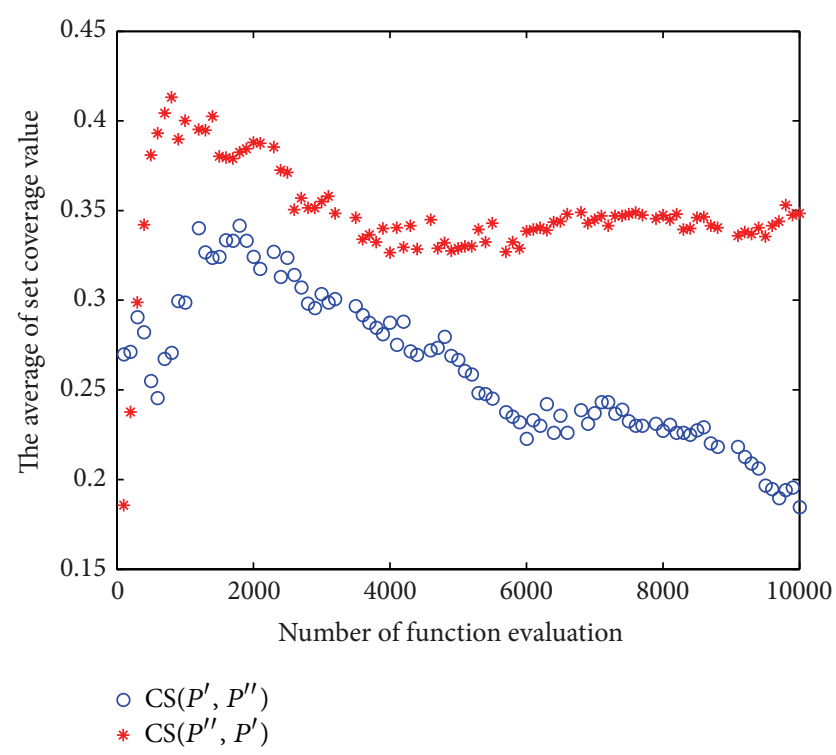

FIGURE 4: Curve of average set coverage value with 10000 function evaluations.

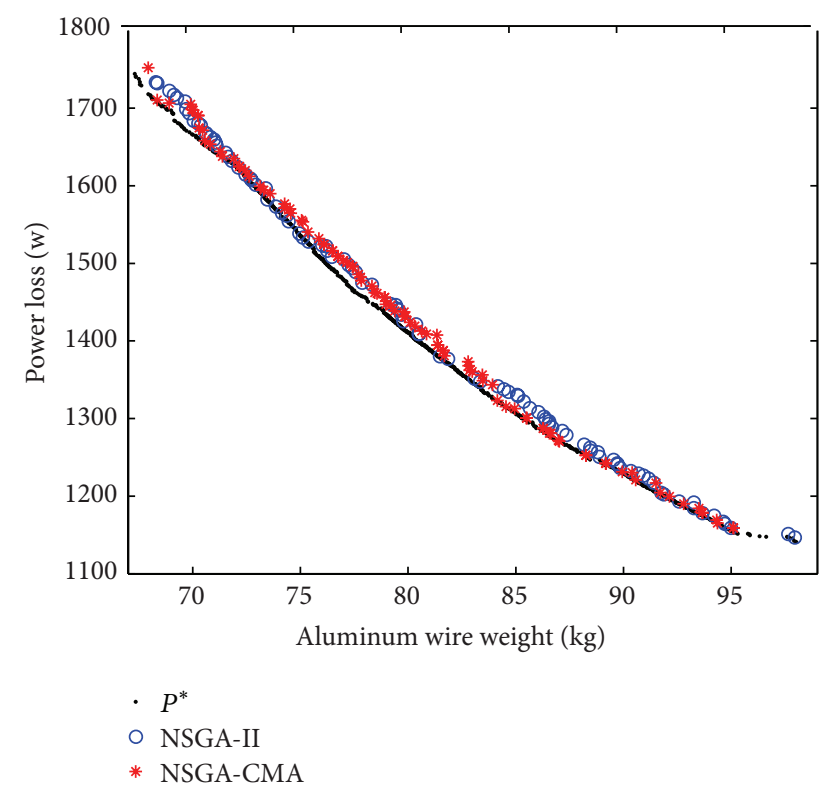

FIGURE 5: Pareto fronts of NSGA-II and NSGA-CMA at the 2500th function evaluation.

optimal solutions obtained by NSGA-CMA are almost the same as the reference set $P^{*}$, however, many solutions among the Pareto optimal solutions obtained by NSGA-II are dominated by the solution in the reference set $P^{*}$. We can conclude that the improved algorithm is more efficiency than the original NSGA-II and it can obtain a better nondominated Pareto front which provides a spectrum of trade-off among the competing objectives.

It can be seen from Table 4 that the aluminum wire weight and the power loss obtained by NSGA-CMA can be reduced at $16.31 \%$ and $20.32 \%$ at most, respectively, compared with

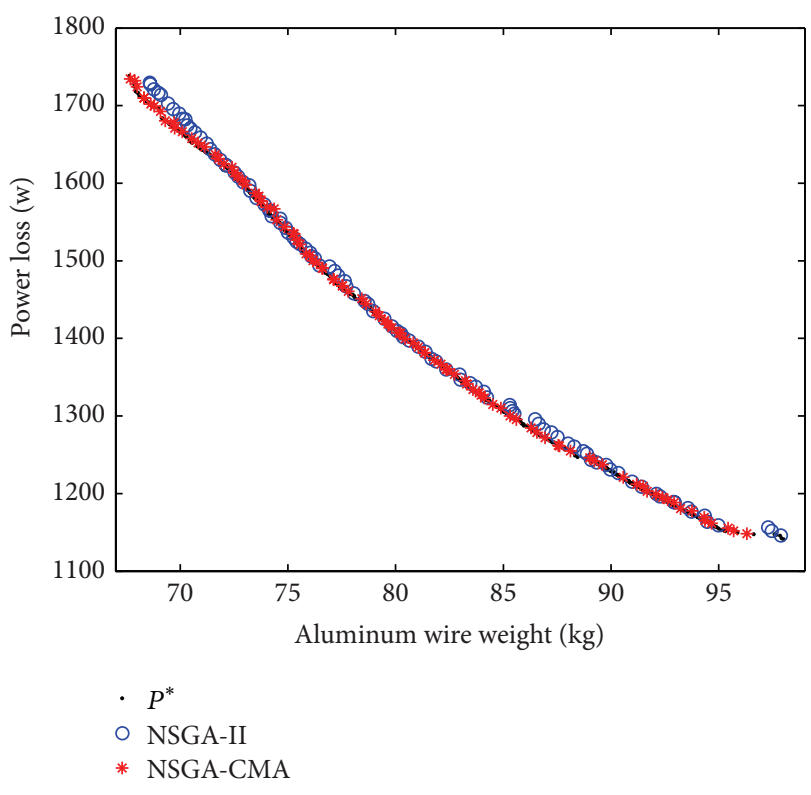

FIGURE 6: Pareto fronts of NSGA-II and NSGA-CMA at the 10000th function evaluation.

TABle 4: A set of Pareto optimal design results.

\begin{tabular}{lcccccc}
\hline Serial number & $N_{p}$ & $N_{c}$ & $D_{\text {in }} / \mathrm{mm}$ & $l_{\text {av }} / \mathrm{mm}$ & $W_{\mathrm{AL}} / \mathrm{kg}$ & $P_{\text {Loss }} / \mathrm{W}$ \\
\hline 1 & 5 & 23 & 570.67 & 2.216 & 69.85 & 1682.38 \\
2 & 6 & 19 & 543.02 & 2.452 & 70.80 & 1665.53 \\
3 & 6 & 18 & 588.00 & 2.538 & 71.64 & 1636.41 \\
4 & 6 & 19 & 597.86 & 2.489 & 72.62 & 1609.73 \\
5 & 6 & 19 & 554.11 & 2.513 & 73.83 & 1574.07 \\
6 & 6 & 19 & 538.23 & 2.532 & 74.71 & 1546.59 \\
7 & 6 & 19 & 592.44 & 2.544 & 75.56 & 1532.94 \\
8 & 6 & 19 & 549.88 & 2.572 & 76.87 & 1492.68 \\
9 & 6 & 19 & 486.59 & 2.586 & 77.41 & 1472.08 \\
10 & 5 & 16 & 599.44 & 2.850 & 78.93 & 1435.91 \\
11 & 5 & 16 & 594.87 & 2.867 & 79.70 & 1417.04 \\
12 & 5 & 16 & 575.60 & 2.889 & 80.88 & 1393.91 \\
13 & 5 & 16 & 535.82 & 2.901 & 81.51 & 1382.52 \\
14 & 5 & 16 & 543.09 & 2.928 & 82.76 & 1351.21 \\
15 & 5 & 16 & 537.47 & 2.936 & 83.15 & 1343.45 \\
\hline
\end{tabular}

the computer aided design result (aluminum wire weight: $83.28 \mathrm{~kg}$, power loss: $1686.00 \mathrm{~W}$ ) [23].

\section{Conclusions}

In this paper, the multiobjective optimization design of drytype air-core reactor made of rectangular cross-section litzwire is studied considering the minimal production cost as well as the minimal operation cost simultaneously. A modified nondominated sorting genetic algorithm-II (called NSGA-CMA) is then proposed to solve this multiobjective optimal problem. Within the NSGA-CMA, the global exploration is done by NSGA-II and the local exploitation by the local search strategy based on CMA-ES to improve the search 
ability and accelerate the convergence speed. In order to guarantee the search efficiency, the local search is applied to nondominated individuals in a given generation and quantity. In addition, an external archive is established for improving the evolutionary efficiency. From the simulation results, it is clearly seen that NSGA-CMA has smaller convergence metric value and more nondominated solutions which dominate the nondominated solutions obtained by NSGA-II. This implies that NSGA-CMA has better search ability and it obtains better Pareto front than NSGA-II. For its promising performance, the NSGA-CMA algorithm is certainly more suitable and effective than NSGA-II for solving the multiobjective design problems of dry-type air-core reactor. Also, it is one of the efficient potential candidates in solving other complicated multiobjective problems.

\section{Conflict of Interests}

The authors declare that there is no conflict of interests regarding the publication of this paper.

\section{References}

[1] S. I. Babic and C. Akyel, "New analytic-numerical solutions for the mutual inductance of two coaxial circular coils with rectangular cross section in air," IEEE Transactions on Magnetics, vol. 42, no. 6, pp. 1661-1669, 2006.

[2] S. I. Babic and C. Akyel, "An improvement in the calculation of the magnetic field for an arbitrary geometry coil with rectangular cross section," International Journal of Numerical Modelling: Electronic Networks, Devices and Fields, vol. 18, no. 6, pp. 493504, 2005.

[3] J. Morozionkov and J. A. Virbalis, "Magnetic field of power plant air core reactor," Elektronika Ir Elektrotechnika, vol. 79, no. 7, pp. 67-70, 2007.

[4] B. S. Ram, "Inductance and winding eddy loss of air-cored reactors," IEE Proceedings-Generation Transmission and Distribution, vol. 146, no. 5, pp. 416-420, 1999.

[5] A. A. Dahab, P. E. Burke, and T. H. Fawzi, "A complete model of a single layer air-cored reactor for impulse voltage distribution," IEEE Transactions on Power Delivery, vol. 3, no. 4, pp. 1745-1753, 1988.

[6] S. L. Varricchio and N. H. C. Santiago, "Electrical strength in air core reactors," in Proceedings of the 4th IEEE International Conference on Properties and Application of Dielectric Materials, pp. 876-879, 1994.

[7] J.-L. Lilien, "Acoustic noise generated by air power reactor in open-air substation," European Transactions on Electrical Power, vol. 16, no. 3, pp. 297-310, 2006.

[8] Q. Wang, Q. Chen, and M. Zeng, "A CFD-taguchi combined method for numerical investigation of natural convection cooling performance of air-core reactor with noise reducing cover," Numerical Heat Transfer Part A: Applications, vol. 55, no. 12, pp. 1116-1130, 2009.

[9] Z.-G. Liu, Y.-S. Geng, J.-H. Wang, and S. Ouyang, "Optimum design of dry-type air-core series reactor based on modified adaptive genetic algorithm," Proceedings of the Chinese Society of Electrical Engineering, vol. 23, no. 9, pp. 103-106, 2003.

[10] Y. Zhao, F. Chen, B. Kang, and X. Ma, "Optimum design of drytype air-core reactor based on the additional constraints balance and hybrid genetic algorithm," International Journal of Applied Electromagnetics and Mechanics, vol. 33, no. 1-2, pp. 279-284, 2010.

[11] K. Deb, A. Pratap, S. Agarwal, and T. Meyarivan, "A fast and elitist multiobjective genetic algorithm: NSGA-II," IEEE Transactions on Evolutionary Computation, vol. 6, no. 2, pp. 182-197, 2002.

[12] P. Murugan, S. Kannan, and S. Baskar, "NSGA-II algorithm for multi-objective generation expansion planning problem," Electric Power Systems Research, vol. 79, no. 4, pp. 622-628, 2009.

[13] S. Kannan, S. Baskar, J. D. McCalley, and P. Murugan, "Application of NSGA-II algorithm to generation expansion planning," IEEE Transactions on Power Systems, vol. 24, no. 1, pp. 454-461, 2009.

[14] L. Xuebin, "Study of multi-objective optimization and multiattribute decision-making for economic and environmental power dispatch," Electric Power Systems Research, vol. 79, no. 5, pp. 789-795, 2009.

[15] L. A. Scola, R. H. C. Takahashi, and S. A. A. G. Cerqueira, "Multipurpose water reservoir management: an evolutionary multiobjective optimization approach," Mathematical Problems in Engineering, vol. 2014, Article ID 638259, 14 pages, 2014.

[16] X. Gao, B. Chen, X. He et al., "Multi-objective optimization for the periodic operation of the naphtha pyrolysis process using a new parallel hybrid algorithm combining NSGA-II with SQP," Computers and Chemical Engineering, vol. 32, no. 11, pp. 28012811, 2008.

[17] N. Hansen and A. Ostermeier, "Completely derandomized selfadaptation in evolution strategies," Evolutionary Computation, vol. 9, no. 2, pp. 159-195, 2001.

[18] N. Srinivas and K. Deb, "Multiobjective function optimization using nondominated sorting genetic algorithms," Evolutionary Computation, vol. 2, no. 3, pp. 221-248, 1995.

[19] N. Krasnogor and J. Smith, "A tutorial for competent memetic algorithms: model, taxonomy, and design issues," IEEE Transactions on Evolutionary Computation, vol. 9, no. 5, pp. 474-488, 2005.

[20] H. Ishibuchi, T. Yoshida, and T. Murata, "Balance between genetic search and local search in memetic algorithms for multiobjective permutation flowshop scheduling," IEEE Transactions on Evolutionary Computation, vol. 7, no. 2, pp. 204-223, 2003.

[21] K. Deb and S. Jain, "Running performance metrics for evolutionary multi-objective optimization," Tech. Rep. 2002004, Indian Institute of Technology, Kanpur, India, 2004.

[22] E. Zitzler, K. Deb, and L. Thiele, "Comparison of multiobjective evolutionary algorithms: empirical results," Evolutionary computation, vol. 8, no. 2, pp. 173-195, 2000.

[23] B. Kang, Optimization model of dry-type air-core reactor and optimization design based on hybrid genetic algorithm [M.S. thesis], Xi'an Jiaotong University, Xi'an, China, 2009. 


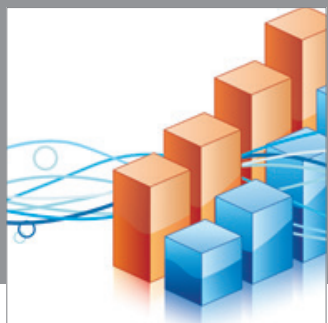

Advances in

Operations Research

mansans

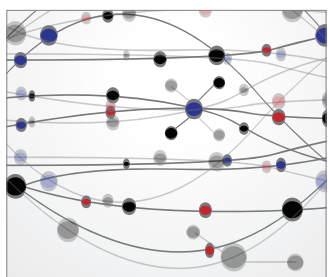

The Scientific World Journal
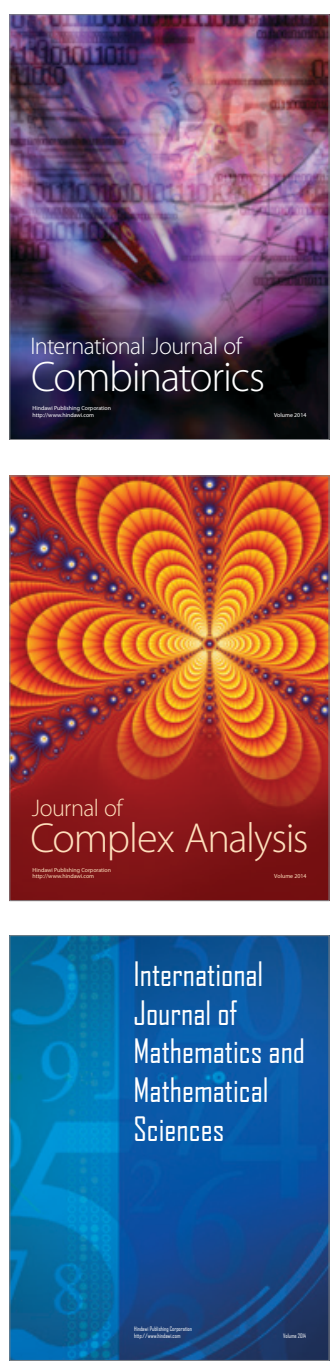
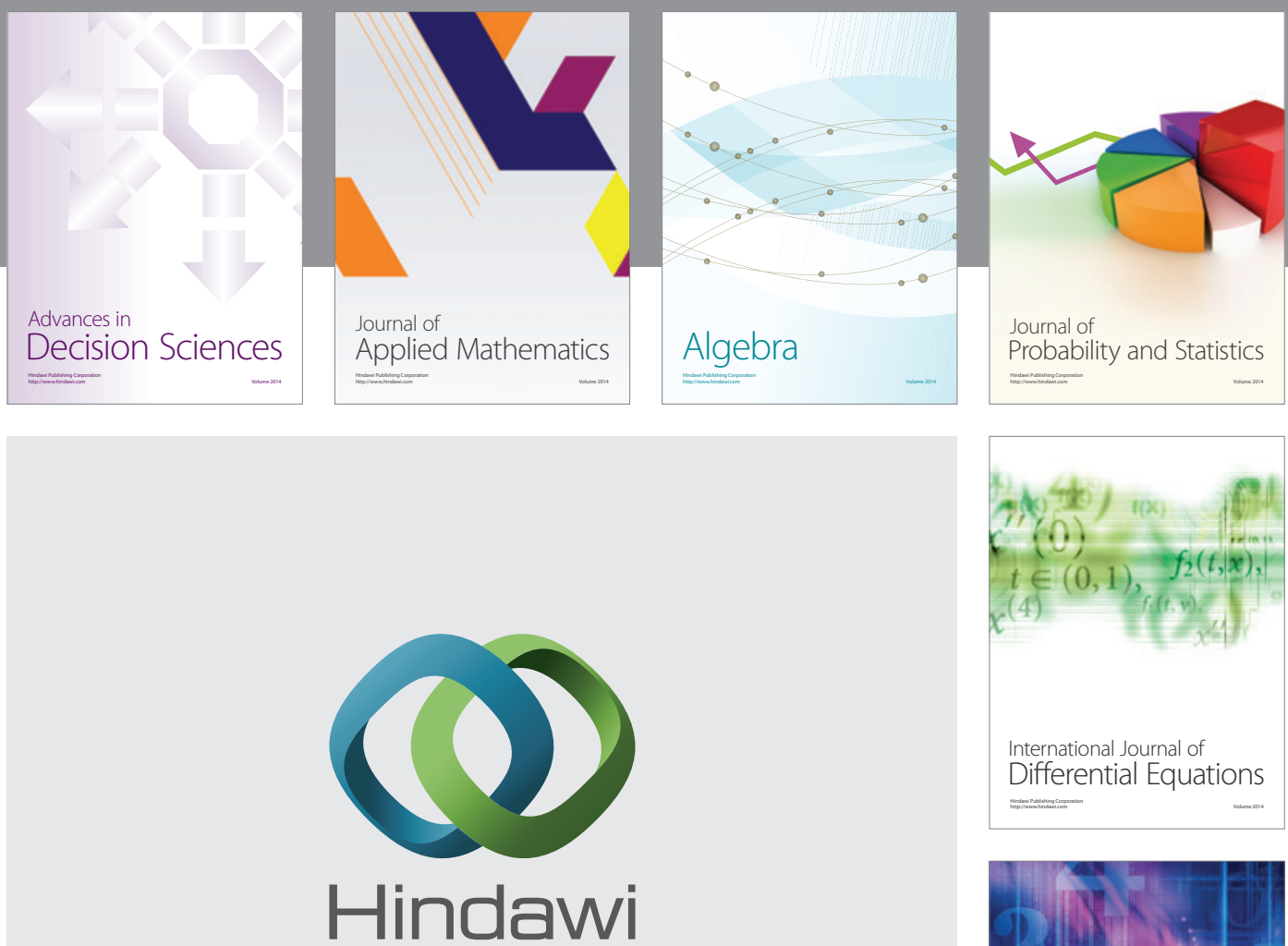

Submit your manuscripts at http://www.hindawi.com
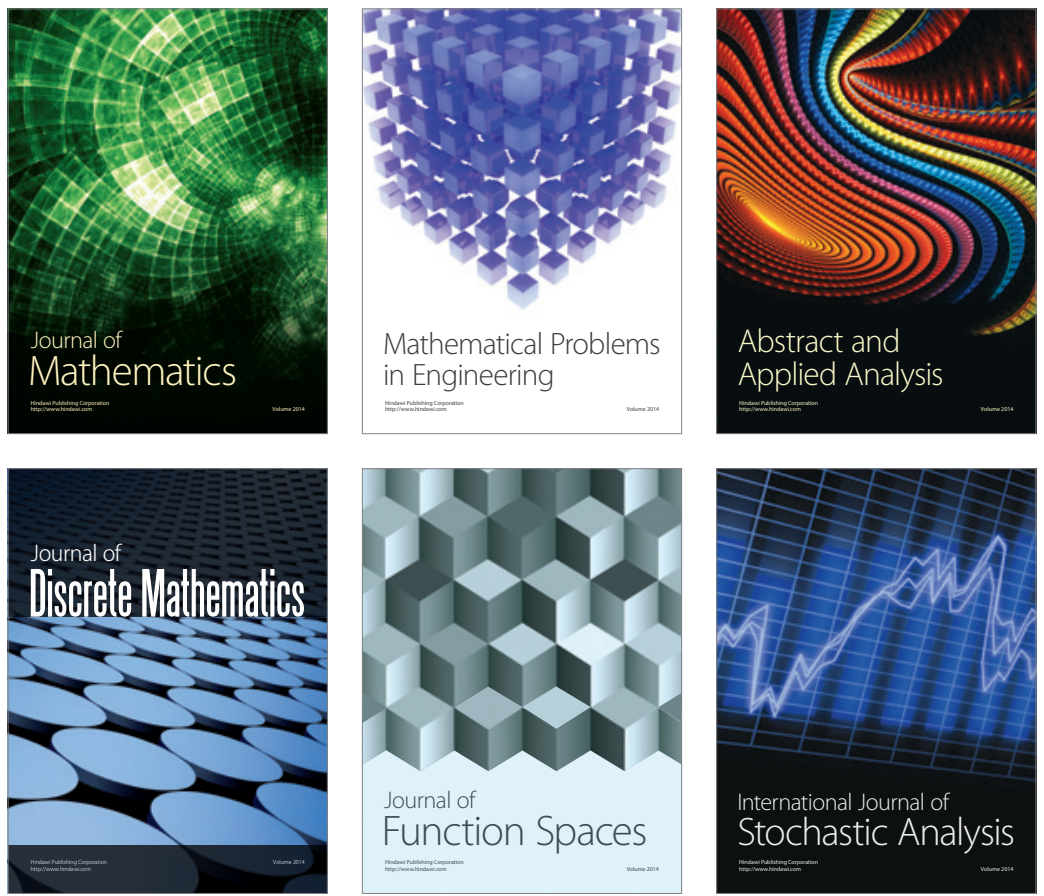

Journal of

Function Spaces

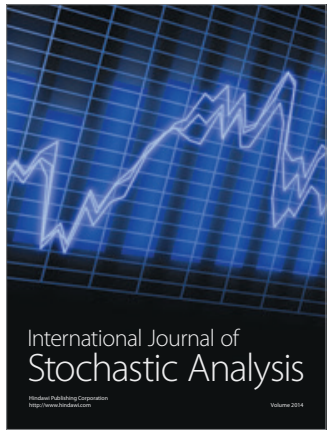

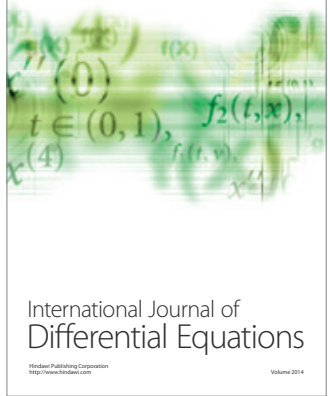
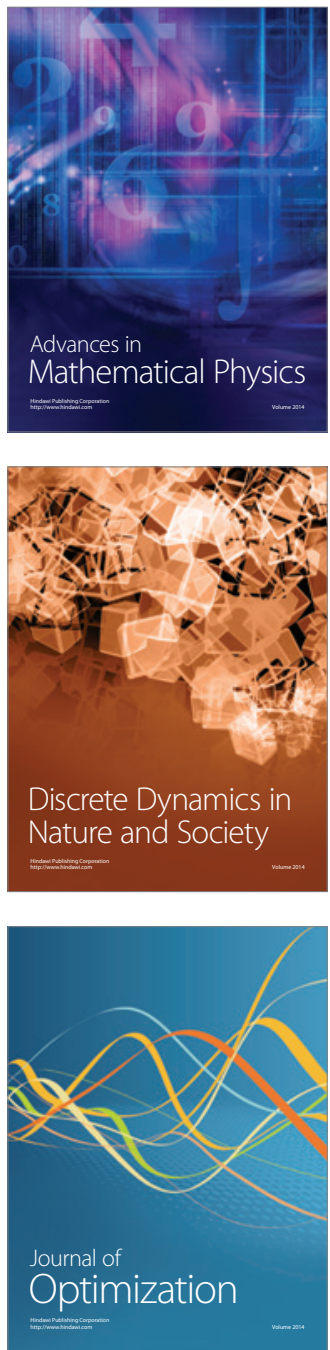